\title{
Numerical Investigation of Flexural Bending in Biaxial Braided Structures for Flexor Tendon Repair
}

\author{
Jerry Ochola1, Benny Malengier², Lieva Van Langenhove ${ }^{2}$ \\ ${ }^{1}$ Department of Manufacturing, Industrial and Textile Engineering, Moi University, Eldoret, Kenya; ${ }^{2}$ Department of \\ Materials, Textiles and Chemical Engineering, Ghent University, Ghent, Belgium
}

Correspondence to: Jerry Ochola, ochollas@gmail.com

Keywords: Braid, Biaxial, Bending, Flexor, Tendons

Received: April 29, $2020 \quad$ Accepted: June 8, $2020 \quad$ Published: June 11, 2020

Copyright $\odot 2020$ by author(s) and Scientific Research Publishing Inc.

This work is licensed under the Creative Commons Attribution International License (CC BY 4.0).

http://creativecommons.org/licenses/by/4.0/

\section{(c) (i) Open Access}

\section{ABSTRACT}

Flexor tendon repair has conventionally been done by suturing techniques. However, in recent times, there have been attempts of using fibrous braided structures for the repair of ruptured tendons. In this regard, the numerical analysis of the flexural stiffness of a braided structure under bending moments is vital for understanding its capabilities in the repair of flexor tendons. In this paper, the bending deflection, curvature, contact stresses and flexural bending stiffness in the braided structure due to bending moments are simulated using Finite Element (FE) techniques. Three dimensional geometry and FE models of five sets of biaxial braided structures were developed using a python programming script. The FE models of the hybrid biaxial braids were imported into ABAQUS (v17) for post-processing and analysis. It was established that the braided fabric with largest braid angle, $\theta=52.5^{\circ}$ had the highest flexural deflection while the lowest deflection was seen in the results of the braided structure with the least braid angle, $\theta=38.5^{\circ}$. The results in this study also portrayed that the curvature in biaxial braids will increase with a decrease in the angle between the braided yarns. This was also consistent with the change of bending angle of the biaxial structures under a bending moment. The deformation of the structures increased with increase in the braid angles. This implies that the flexural bending stiffness decreased with increase in braid angle. The stress limits during bending of the braided structures were established to be within the range that could be handled by flexor tendons during finger bending.

\section{INTRODUCTION}

Flexor tendons are found in the fingers of the human hand (Figure 1(a)) and are crucial in effecting 
the movement of the fingers-a process known as flexion (Figure 1(b)). The tendons therefore undergo bending displacements during normal use.

Braided structures have been used in diverse fields and for myriad applications [1-3]. In the medical fields, stents are mainly used for support in annular structures and their potential in flexor tendon repair has been explored, $[4,5]$. In order to develop a braided structure suitable for possible application in tendon repair, flexural bending is a crucial deformation parameter that could be considered.

The analysis of the mechanical deformation of braided structures has been performed using mathematical models [6] which have provided a useful tool for predicting geometrical and mechanical properties of the structures [7]. Generalized algorithms have been used to calculate interlacing points of braids both for biaxial and triaxial braids [8]. Geometrical modeling techniques for biaxial braided structures have also been based on the generalized rose curve [9]. However, most studies carried out so far focused mainly on the failure and deformation of braided structures due to transverse and longitudinal loading [10].

The aim of this study was to investigate the bending of biaxial braided structures in their potential use in tendon repair. In this context therefore, the flexural bending and stress distribution under a moment which may occur due to finger flexion were investigated based on finite element methods. 3D models of hybrid biaxial configurations of braided structures were developed and analyzed. Stress distributions at the yarn-yarn interface are obtained based on the numerical and analytical results.

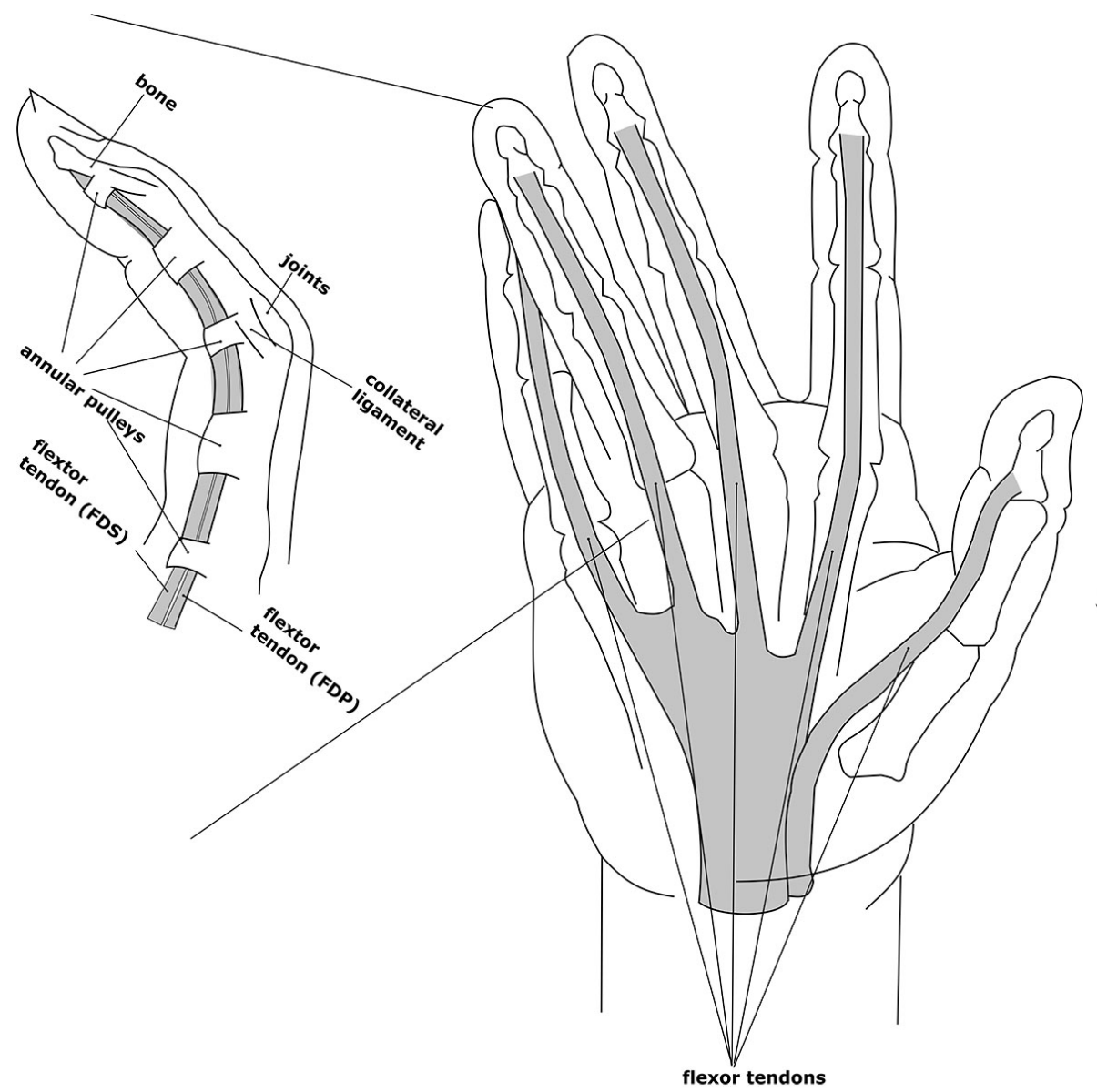

(a)

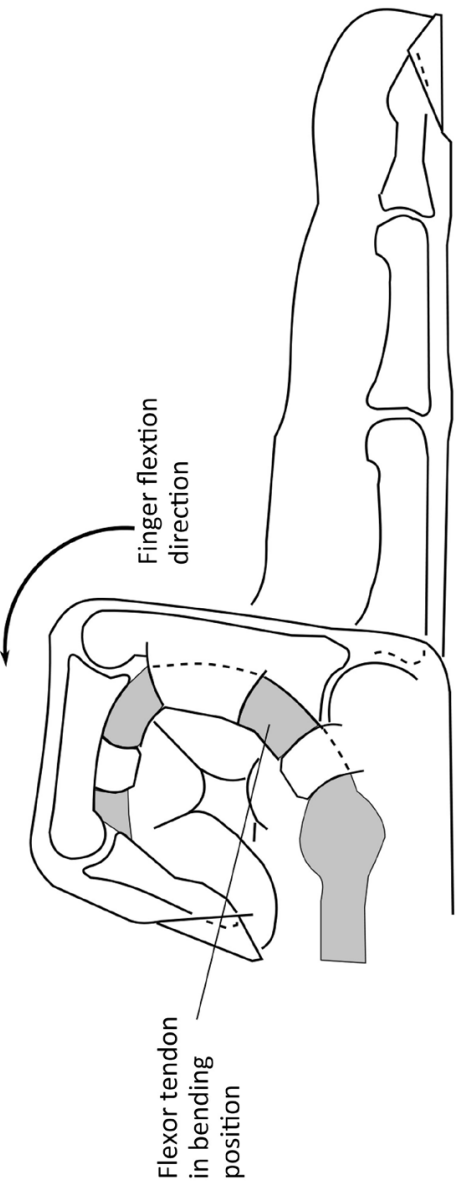

(b)

Figure 1. (a) Anatomy showing position of the flexor tendons in the human hand with the side view (top-left) indicating pulleys holding the tendons in a curved configuration during finger flexion. (b) The flexion action of a human finger showing the deformation of the tendons when the finger bends. 


\section{NUMERICAL MODELING AND MATERIAL PROPERTIES}

\subsection{Construction of the Braid Geometry}

The geometrical model of the biaxial braided structures were developed using a pre-processor (pFormex 0.9.0), [11] based on a python script using the parameters shown in Table 1. The material for the yarns was considered as PHYNOX. A hybrid braided structure was developed by expanding the original python script [11] for a braided structure using mathematical transformations to create multiple yarns in the clockwise (NE) and anti-clockwise (SE) directions by implementing the dimensions shown in Figure 2. The FE model of the tubular braided structure (Figure 3) was developed by meshing the yarns using Timoshenko beam elements (B31). The FE models were then imported into ABAQUS (v17) for analysis and pre-processing. Due to the complexity of the extra yarn in the clockwise and anti-clockwise directions 5 sets of braid angles $(\theta)$ were considered for the braided structures for faster computation time, these were: $38.5^{\circ}, 45^{\circ}, 48.5^{\circ}$ and $52.5^{\circ}$.

Table 1. Parameters for braided structure 3D Model.

\begin{tabular}{ccc}
\hline Parameter & Description & Value \\
\hline$D(\mathrm{~mm})$ & initial nominal diameter & 2 \\
$d(\mathrm{~mm})$ & yarn diameter & 0.06 \\
$n(-)$ & total number of yarns & 16 \\
$L(\mathrm{~mm})$ & initial nominal length & 10 \\
$E(\mathrm{mpa})$ & Young's modulus & 206,000 \\
$\mu(-)$ & friction & 0.2 \\
$Y(\mathrm{~N})$ & yield strength & 2450 \\
\hline
\end{tabular}

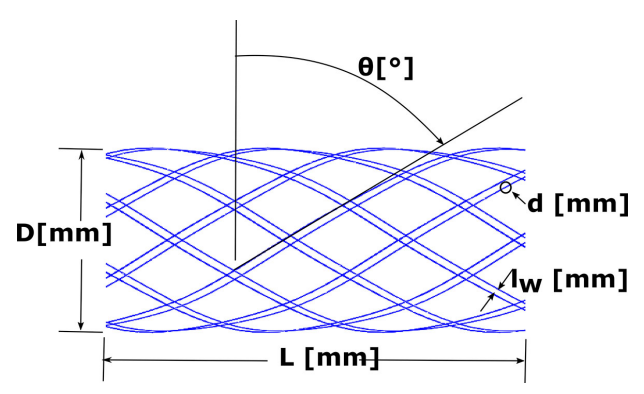

Figure 2. Geometrical parameters of the biaxial braided structure.

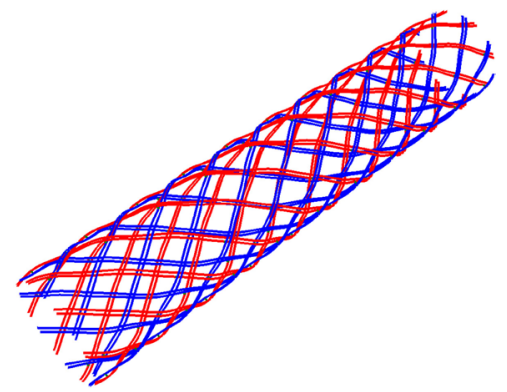

Figure 3. Finite element geometry of the biaxial braided structure for a 16 yarn structure showing nominal longitudinal length $\left(L_{o}=10 \mathrm{~mm}\right)$, nominal diameter $\left(D_{e}=2 \mathrm{~mm}\right)$ and the braid angle, $\theta=52.5^{\circ}$. 


\subsection{Boundary Conditions and Loading}

The finite element model of the biaxial braided structure (hybrid) created in pyFormex was imported into ABAQUS (v17). The flexural bending moment was induced by a moment of $1.5 \mathrm{~N} \cdot \mathrm{mm}$ using a smooth step amplitude at $0.01 \mathrm{~s}$ at one end of the braided structure while the proximal end of the structure was fixed by constraining all displacements and rotations. This was done using the following steps:

- $\quad$ symmetrical boundary conditions applied to all nodes at one of of the braided structure to constrain the displacement and rotation;

$$
u_{1}=0, u_{2}=0, u_{3}=0, u r_{1}=0, u r_{2}=0, u r_{3}=0
$$

- the nodes at the opposite end of the braided structure are constrained to a reference point (RP) using an MPC constraint and a moment load applied to the RP.

These boundary conditions mimic the actual flexor tendon bending behaviour during finger movement during flexion as illustrated in Figure 4(b).

\subsection{Numerical Analysis}

The numerical analysis of the flexural bending stiffness was performed using beam theory, which implies that a braided structure can be regarded as a beam [12]. When a bending moment is applied on the braided structure, it will undergo a rotation which is equivalent to a bending angle $(\alpha)$ and a flexural bending stiffness $E I$ which can be evaluated as shown in Equation (1):

$$
E I=\frac{m L}{2 \alpha}
$$

The flexural bending deformation of the braided structure can also be computed at different curvatures [12] as illustrated in Figure 4(b). In this case the bending stiffness can be formulated as shown in Equation (2).

$$
E I=m \rho
$$

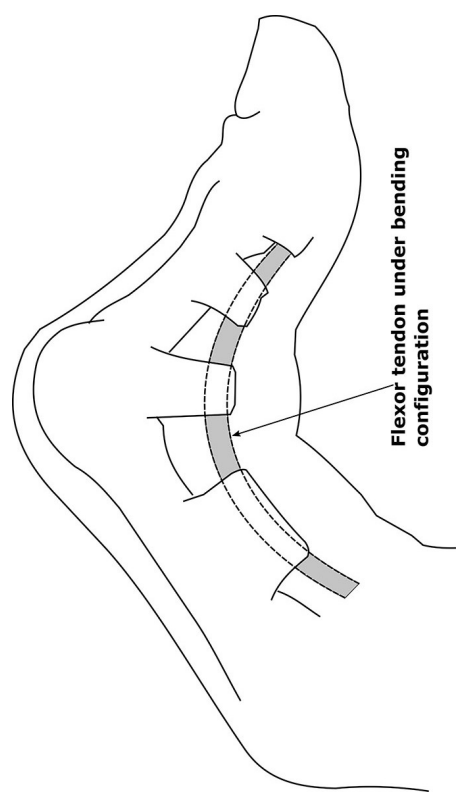

(a)

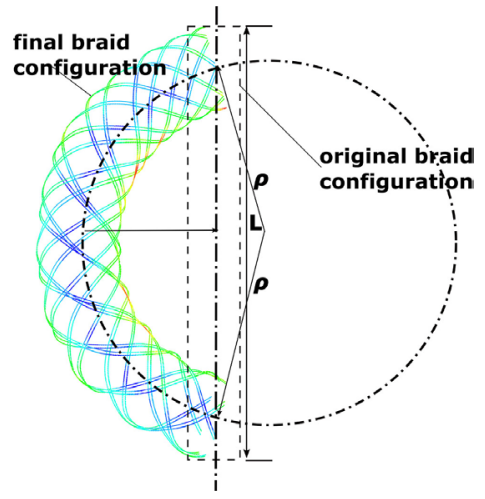

(b)

Figure 4. (a) An illustration of a flexor tendon in a bending configuration due to a potential bending load. (b) Schematic illustration of a biaxial braided structure model under the bending moment $(m)$, where $v$ is the bending deflection and $\rho$ is the radius of curvature $[12,13]$. 
where the curvature of the braided structure under the bending moment, $m$ can be evaluated as:

$$
\frac{1}{\rho}=\frac{m}{E I}
$$

The mathematically evaluated flexural stiffness was used to verify the FEA data from the bending simulations by computing root mean square error (RMSE) (Equation (4)) and mean absolute percentage error (MAPE) (Equation (5)) between analytical data and the FE data.

$$
\begin{gathered}
\mathrm{RMSE}=\sqrt{\frac{1}{N} \sum_{n=0}^{N-1}\left(y_{a}(n)-y_{i}(n)\right)^{2}} \\
\mathrm{MAPE}=\frac{1}{n} \sum\left|\frac{y_{a}-y_{i}}{y_{a}}\right|
\end{gathered}
$$

where $y_{a}=$ analytical data, $y_{i}=$ FE data.

\section{RESULTS AND DISCUSSIONS}

\subsection{Effects of Bending Moment on Flexural Deflection}

In order to investigate the effects of bending moment on flexural deflection of the braided structure, 5 numerical models of the biaxial braided structure were developed by varying the braid angle. The results of the evolution of the bending moment with deflection are illustrated by the curves in Figure 5(a). The curves for the change in bending moment with the deflection of the braided structures shows an increase in deflection with increasing moment for most parts of the curves. It can be seen that when the braided fabric with largest braid angle, $\theta=52.5^{\circ}$ had the highest flexural deflection while the lowest deflection was seen in the results of the braided structure with the lowest braid angle, $\theta=38.5^{\circ}$.

The deflection in the braided structure with $\theta=38.5^{\circ}$ as observed to decrease after bending moment of $1 \mathrm{~N} \cdot \mathrm{mm}$. This was probably due to the cover factor in the structure which caused more yarn-yarn displacement within the structure. This is also illustrated by the drop in the contact stress at this point within the structure as shown in Figure 6(b) and also by the contour plots in Figure 7.

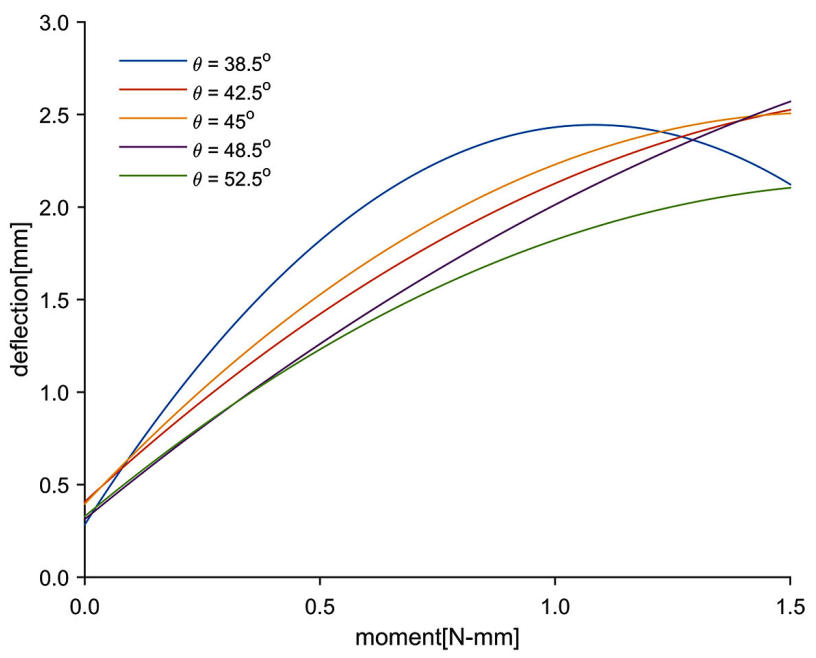

(a)

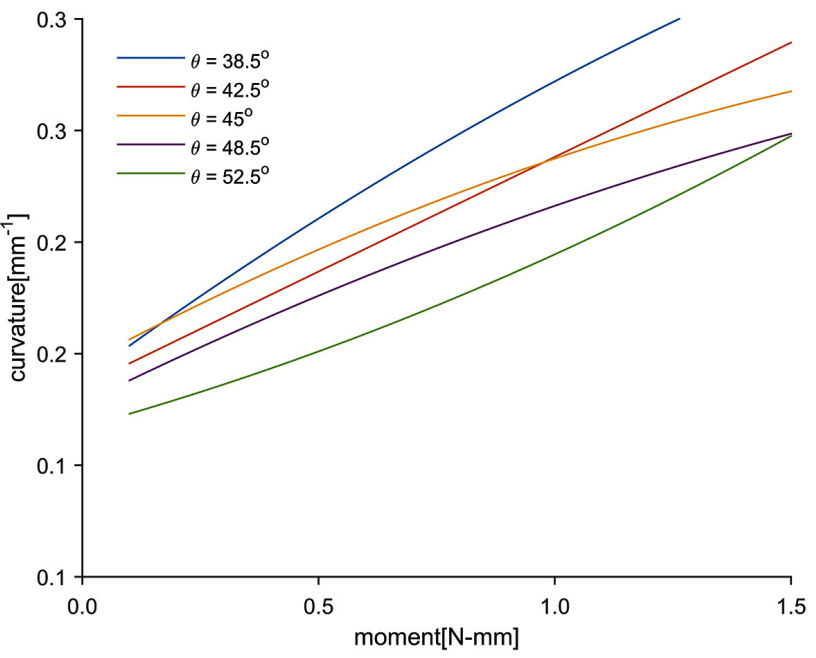

(b)

Figure 5. Comparison of the flexural bending properties of the braided structure showing: (a) moment-bending deflection curves to the changes in braided angle in the deformed configuration and (b) moment-curvature curves relative to the changes in braided angle in the deformed configuration. 


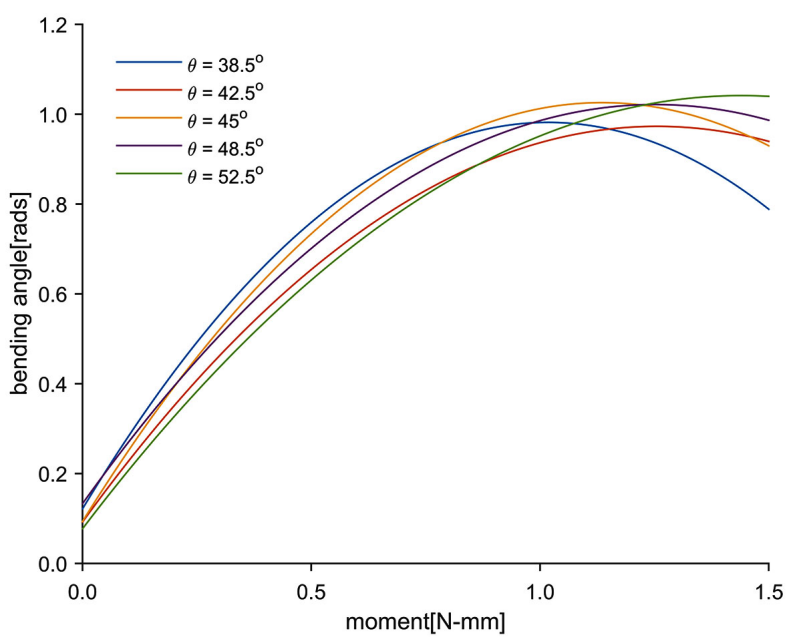

(a)

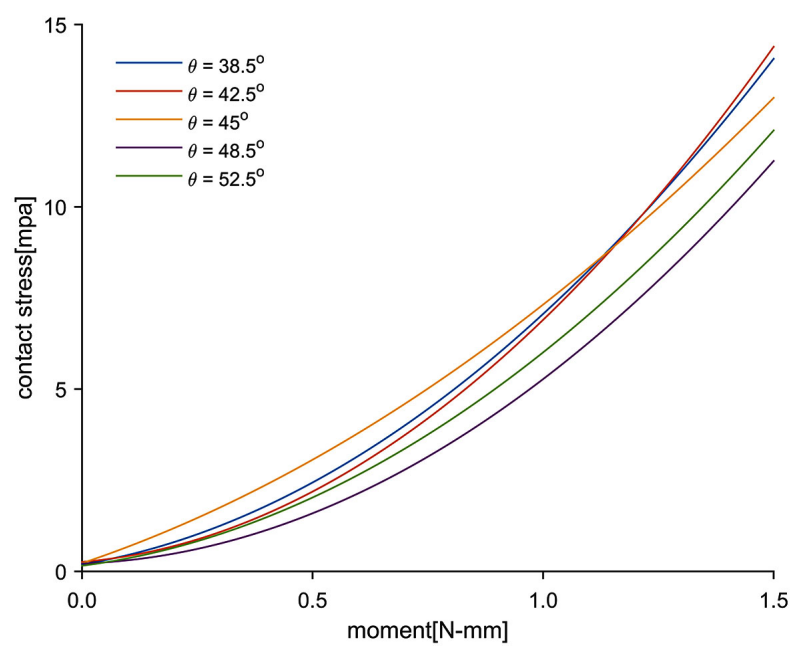

(b)

Figure 6. Comparison of the flexural bending properties of the braided structure: (a) moment-bending angle curves to the changes in braided angle in the deformed configuration and (b) moment-contact stress curves relative to the changes in braided angle in the deformed configuration.

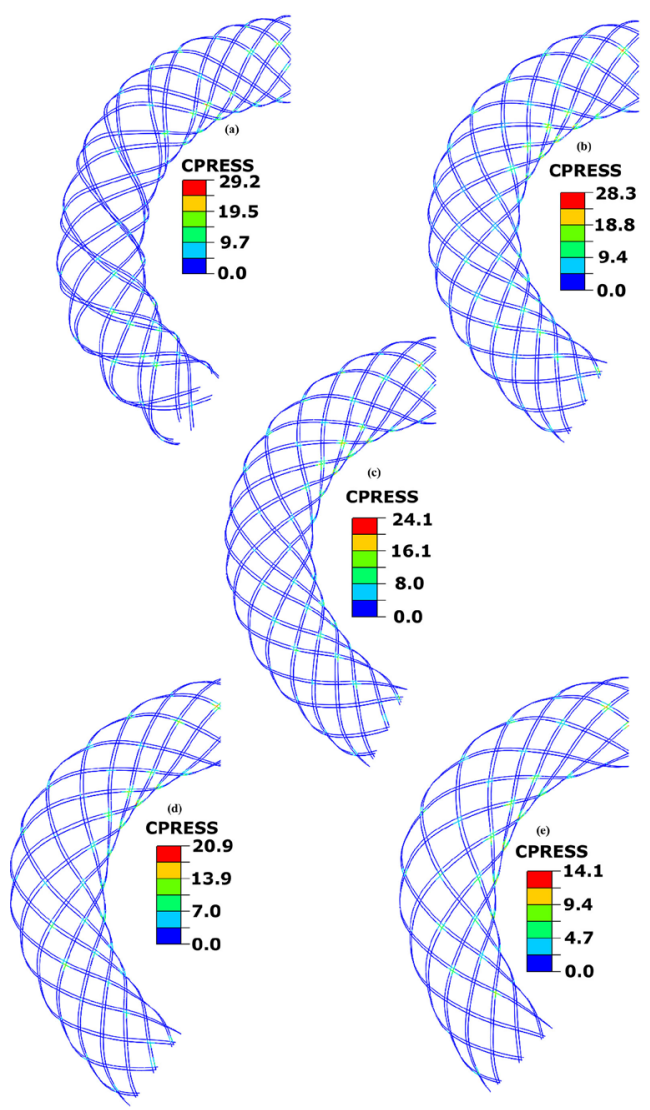

Figure 7. Contour plots of contact stresses for the braided structure in deformed configuration under a bending moment for (a) braid angle; $\theta=38.5^{\circ}$, (b) braid angle; $\theta=42.5^{\circ}$, (c) braid angle; $\theta=45^{\circ}$, (d) braid angle; $\theta=48.5^{\circ}$, (e) braid angle; $\theta=52.5^{\circ}$. 


\subsection{Effects of Bending Moment on Braid Curvature}

The analysis results of the change in the bending moment with curvature are illustrated by the curves in Figure 5(b). The results indicate near linear increase between the bending moment and curvature. The braided structure with angle of $\theta=38.5^{\circ}$ showed they had the highest curvature while $\theta=52.5^{\circ}$ had the least curvature of the sets of braided structures considered in this study. This indicates that the curvature of the biaxial braided structures increased with decrease in the braided angle.

\subsection{Effects of Moment on Bending Angle}

The results for the bending angle of the braided structure with change in the bending moment are shown in Figure 6(a). The results illustrate an increase of the bending angle with increase bending moment for most parts of the curves. There was also evidence from the data that as the braided angle increase the bending angle of the biaxial structures decreased; this was portrayed by the higher rate of increase of the $\theta=38.5^{\circ}$ braided structure as compared to the $\theta=52.5^{\circ}$ structure.

\subsection{Effects of Bending Moment on Contact Stress}

The analysis results of the change in the bending moment with contact stress at the yarb-yarn interface is illustrated by the curves in Figure 6(b), which also shows deformation as a result of the applied bending moment for the biaxial braided structure models. It was established from the results that the contact stress in the braided structure with a braid angle of $\theta=52.5^{\circ}$, was the lowest while the structure with a braid angle $\theta=38.5^{\circ}$ had the highest contact stress of the 5 models considered in this study. The bending moment was applied to the model uniformly. The deformation of the $\theta=38.5^{\circ}$ braid was also more than that of the $\theta=52.5^{\circ}$ braided structure.

\subsection{Verification of FE Models}

The numerical results of the bending stiffness with increasing bending moment are shown in Figure 8(a). The data plotted shows the bending stiffness of the biaxial braided structures considered in this study decreased with increase in braid angle (from $\theta=38.5^{\circ}$ to $\theta=52.5^{\circ}$ ).

The verification of the FE models used in this study was performed by evaluating the bending stiffness of the biaxial braided structure at $\theta=52.5^{\circ}$ using the beam theory approach proposed in previous research [12]. This is illustrated by the curves of change in bending moment with bending stiffness shown in Figure 8(b). The results show that the analytical equation can be used to predict the flexural bending stiffness of biaxial braided structures as shown by the close conformity of the profiles of the curves (Figure 8(b)). This was established using the RMSE (4) and MAPE (5). The data indicated that the $\theta=52.5^{\circ}$ structure had a error margin with analytical data of $\mathrm{RMSE}=0.565$, and $\mathrm{MAPE}=-0.038$, this implies that the data had a better fit with the analytical data $(\mathrm{RMSE}=0.565)$ and also a better forecast ( $\mathrm{MAPE}=-0.038)$ of the models considered in this study.

\section{CONCLUSION}

Numerical models for a biaxial braided structure were developed in this study. Five sets of the structure were developed by varying the braided angle in terms of $\theta=38.5^{\circ}, \theta=42.5^{\circ}, \theta=45^{\circ}, \theta=48.5^{\circ}$ and $\theta=52.5^{\circ}$. A bending moment was applied on each of the models to mimic the flexing of a human finger and an FEA method used to investigate the flexural bending properties of the biaxial braids. It can be seen that when the braided fabric with largest braid angle $\left(\theta=52.5^{\circ}\right)$ had the highest flexural deflection while the lowest deflection was seen in the results of the braided structure with the least braid angle $\left(\theta=38.5^{\circ}\right)$. The results in this study portrayed the curvature in biaxial braids will increase when the decrease in the angle between the braided yarns. This was also consistent with the bending angle of the biaxial structures under a bending moment which suggested that the deformation was as a result of the applied 

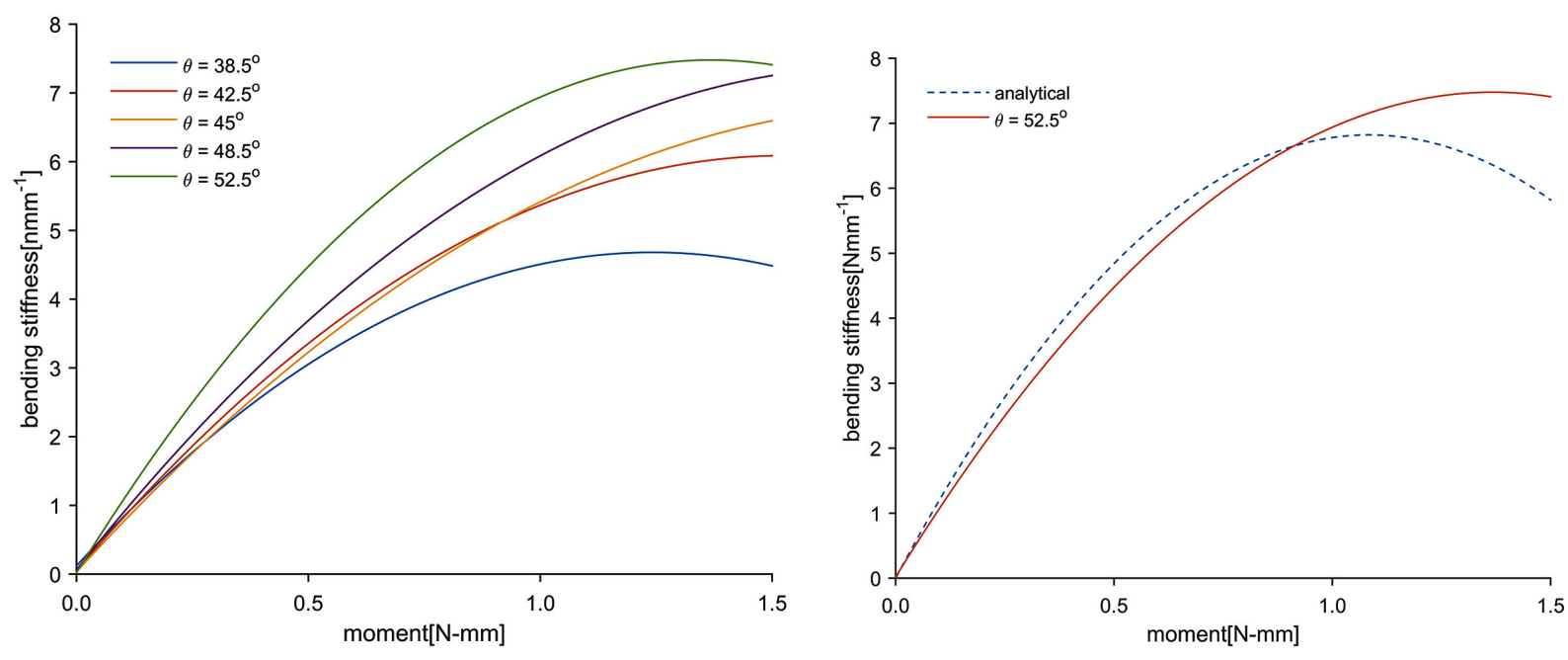

Figure 8. (a) Moment-FE bending stiffness curves relative to the changes in braided angle in the deformed configuration at $t=0 \mathrm{~s}$ to $t=0.06 \mathrm{~s}$, (b) verification of the FE data using beam theory approach showing moment-analytical bending stiffness alongside FE bending stiffness curves relative to the changes in braided angle in the deformed configuration at $t=0 \mathrm{~s}$ to $\mathrm{t}=0.06 \mathrm{~s}$.

bending moment. Therefore, the deformation of the structures increased with increase in the braid angles. In this regard, the numerical results of the bending stiffness with increasing bending moment decreased with increase in braid angle.

\section{CONFLICTS OF INTEREST}

The authors declare no conflicts of interest regarding the publication of this paper.

\section{REFERENCES}

1. Irsale, S. and Adanur, S. (2006) Design and Characterization of Polymeric Stents. Journal of Industrial Textiles, 35, 189-200. https://doi.org/10.1177/1528083706055752

2. Noorbakhsh, M., Rowshanzamir, M., Mahdi, A.S. and Mahdi, H.S. (2019) An Experimental Investigation into the Behavior of Sand Reinforced by Tubular Braided Structures. Journal of Industrial Textiles. https://doi.org/10.1177/1528083719866940

3. Liu, G.H., Hu, H., Zhang, P.H. and Wang, W.Z. (2006) Radial Compressive Properties of the Biodegradable Braided Regeneration Tubes for Peripheral Nerve Repair. Journal of Industrial Textile, 36, No. 35. https://doi.org/10.1177/1528083706064375

4. Mathew, B. (2013) Jan. 10. Tendon Repair Device Method. United States Patent US 2013/0013065A1.

5. Ochola, J., Malengier, B., Daelemans, L., Githaiga, J. and Van Langenhove, L. (2018) Experimental and Numerical Analysis of the Tendon Repair Process Using Tubular Braided Fabrics. Autex Research Journal, 18, 121-129. https://biblio.ugent.be/publication/8568511/file/8568512.pdf https://doi.org/10.1515/aut-2017-0007

6. Rawal, A., Potluri, P. and Steele, C. (005) Geometrical Modeling of the Yarn Paths in Three-Dimensional Braided Structures. Journal of Industrial Textiles, 35, No. 2. https://doi.org/10.1177/1528083705057574

7. Michael, R.J. and Claude, O.C. (1993) A Study of the Geometrical and Mechanical Properties of a Self-Expanding Metallic Stent-Theory and Experiment. Journal of Applied Biomaterials, 4, 77-85. 
https://doi.org/10.1002/jab.770040111

8. Kyosev, Y. (2019) Topology Based Models of Tubular and Flat Braided Structures. In: Topology-Based Modeling of Textile Structures and Their Joint Assemblies. Springer, Cham.

https://doi.org/10.1007/978-3-030-02541-0

9. Ning, F., Potluri, P., Yu, W. and Hearle, J. (2017) Geometrical Modeling of Tubular Braided Structures Using Generalized Rose Curve. Textile Research Journal, 87, 474-486. https://doi.org/10.1177/0040517516632471

10. Liu, Y.S. and Kyosev, Y. (2019) Automatic Analysis the Braiding Angle of the Braided Fabrics Using Image Processing. Fibres and Textiles, 26, 63-68.

11. pyFormex Example Scripts - Wire Stent. (2020) http://www.nongnu.org/pyformex/doc-0.9/examples.html

12. Ni, X.-Y., Pan, C.-W. and Gangadhara, P.B. (2015) Numerical Investigations of the Mechanical Properties of a Braided Non-Vascular Stent Design Using Finite Element Method. Computer Methods in Biomechanics and Biomedical Engineering, 18, 1117-1125. https://doi.org/10.1080/10255842.2013.873420

13. Wu, W., Yang, D.-Z., Qi, M. and Wang, W.-Q. (2007) An FEA Method to Study Flexibility of Expanded Coronary Stents. Journal of Materials Processing Technology, 184, 447-450.

https://doi.org/10.1016/j.jmatprotec.2006.12.010 